\title{
Hubble Parameter Measurement Constraints on Holo- graphic Dark Energy
}

\author{
Plato Lum You Sheng ${ }^{1, *}$ and Cindy Ng Shao Chin ${ }^{1, * *}$ \\ ${ }^{1}$ National University of Singapore, Faculty of Science, Department of Physics, 2 Science Drive 3, Sin- \\ gapore 117551.
}

\begin{abstract}
We review and attempt to constrain the $\Lambda$ CDM and XCDM models using 26 Hubble parameter against redshift data points from [1], [2], [3], [4] and [5]. Also known as the cosmic chronometers approach, these constraints are now almost as restrictive as or less than those from Type 1a Supernova (SN1a) apparent magnitude versus redshift data [6]. Then, we attempt to constrain the Holographic Dark Energy model [7] using the aforementioned $26 \mathrm{H}(z)$ measurement. Although interesting, errors for the $H(z)$ could be less well estimated as it is a relatively new measure relative to $\mathrm{SN} 1 \mathrm{a}$ data, which has been studied for a much longer time.
\end{abstract}

\section{Main Objectives}

In this research, we attempt to test the $\Lambda \mathrm{CDM}, \mathrm{XCDM}$, and Holographic Dark Energy models with the Hubble parameter measurement using a modified $\chi^{2}$ test to ascertain which of the three above best matches the observational data. This modified $\chi^{2}$ test involves the use of a posterior likelihood function to remove any dependence on the nuisance parameters.

\section{$2 \Lambda$ CDM Model}

The standard model of cosmology is the spatially-flat $\Lambda$ CDM model [8]. Roughly $73 \%$ of the energy budget in this model is dark energy, represented by $\Lambda$, more famously known as Einstein's cosmological constant. Cold Dark Matter (CDM) makes up of majority of the remaining energy budget. The rest is made up of non-relativistic baryonic matter. The dark energy density is constant in time and space.

Even though most predictions made from the $\Lambda \mathrm{CDM}$ model align reasonably with experiments, there are some inconsistencies, such as the coincidence puzzle. In this model,

$$
H^{2}(z)=H_{0}^{2}\left[\Omega_{m 0}(1+z)^{3}+\Omega_{\Lambda}+\left(1-\Omega_{m 0}-\Omega_{\Lambda}\right)(1+z)^{2}\right]
$$

\footnotetext{
*e-mail: a0124019@u.nus.edu

**e-mail: cindy.ng@nus.edu.sg
} 


\section{XCDM Model}

We use a parametrization, known as XCDM, to describe slowly changing dark energy density, decreasing for quintessence, increasing for phantom energy. In XCDM, dark energy is modeled as a spatially homogeneous $X$-fluid with an equation of state $p_{X}=w_{X} \rho_{X}$. The parameter $w_{X}<-\frac{1}{3}$ is independent of time. $p_{X}$ and $\rho_{X}$ are the pressure and energy density of the X-fluid. When $w_{X}=-1$, the XCDM model reduces to the $\Lambda$ CDM model. However, the XCDM model is incomplete as it is unable to describe spatial inhomogeneties [9]. For computational simplicity, we assume a spatially flat XCDM model. In this model,

$$
\begin{gathered}
\rho_{X} \propto a^{-3\left(1+\omega_{X}\right)}, \\
H^{2}(z)=H_{0}^{2}\left[\Omega_{m 0}(1+z)^{3}+\left(1-\Omega_{m 0}\right)(1+z)^{3\left(1+\omega_{X}\right)}\right] .
\end{gathered}
$$

\section{Holographic Dark Energy}

This model arises from a theoretical attempt of applying the holographic principle to dark energy [7]. As the holographic principle originates from string theory, it is hoped that the successful application of the holographic principle to dark energy would translate into quantum gravity, thus allowing for further unification in physics. Basically, the holographic principle proposes that any information regarding an $N$ dimensional volume can be encoded on the surface of $N-1$ dimenions on its boundary [10]. For example, all information regarding a 3D sphere can be gleaned from just studying its 2D surface. As recent observations have shown that the universe is flat, we consider only a flat Universe when constraining dark energy with the Holographic model. In this model

$$
\begin{gathered}
H^{2}(z)=H_{0}^{2}\left[\Omega_{d e 0} X(z)+\left(1-\Omega_{d e 0}\right)(1+z)^{3}\right], \\
\frac{d \Omega_{d e}}{d z}=-\frac{\Omega_{d e}\left(1-\Omega_{d e}\right)}{1+z}\left(1+\frac{2 \sqrt{\Omega_{d e}}}{C}\right), \\
X(z)=\exp \left[3 \int_{0}^{z} \frac{\left(1+w\left(z^{\prime}\right)\right)}{1+z^{\prime}} \mathrm{d} z^{\prime}\right] \\
w=-\frac{1}{3}\left(1+\frac{2 \sqrt{\Omega_{d e}}}{C}\right) .
\end{gathered}
$$

\section{$5 \chi^{2}$ Test}

Using the $\chi^{2}$ test, we attempt to constrain the various aforementioned models;

$$
\chi_{H}^{2}\left(H_{0}, p\right)=\sum_{i=1}^{26} \frac{\left[H_{t h}\left(z_{i} ; H_{0}, \mathbf{p}\right)-H_{o b s}\left(z_{i}\right)\right]^{2}}{\sigma_{i}{ }^{2}}
$$

where $H_{t h}\left(z_{i} ; H_{0}, \mathbf{p}\right)$ is the theoretical value of the Hubble parameter predicted by the model and $H_{o b s}\left(z_{i}\right)$ is the observed value of the Hubble parameter. To remove the nuisance parameter $H_{0}$, we assume $H_{0}$ has a Gaussian distribution with one standard deviation $\sigma_{H_{0}}$ and mean $\bar{H}_{0}$. We then marginalize over $H_{0}$ with a posterior likelihood function $\mathcal{L}_{H}(p)$ that only depends 
on the model parameters, $\mathbf{p}$, by integrating the product of $e^{-\chi_{H}^{2} / 2}$ and the $H_{0}$ prior likelihood function $e^{-\left(H_{0}-\bar{H}_{0}\right)^{2} / 2 \sigma_{H_{0}}^{2}}[11]$. We define

$$
\begin{gathered}
\alpha=\frac{1}{\sigma_{H_{0}}^{2}}+\sum_{i=1}^{26} \frac{E^{2}\left(z_{i} ; \mathbf{p}\right)}{\sigma_{i}^{2}}, \beta=\frac{\bar{H}_{0}}{\sigma_{H_{0}}^{2}}+\sum_{i=1}^{26} \frac{H_{o b s}\left(z_{i}\right) E\left(z_{i} ; \mathbf{p}\right)}{\sigma_{i}^{2}}, \gamma=\frac{\bar{H}_{0}^{2}}{\sigma_{H_{0}}^{2}}+\sum_{i=1}^{26} \frac{H_{o b s}{ }^{2}\left(z_{i}\right)}{\sigma_{i}^{2}}, \\
\operatorname{erf}(x)=\frac{2}{\sqrt{\pi}} \int_{0}^{x} e^{-t^{2}} \mathrm{~d} t
\end{gathered}
$$

where $E=H_{t h} / H_{0}$. Thus, the integral can be expressed in terms of the error function (erf), as follows:

$$
\mathcal{L}_{H}(p)=\frac{1}{2 \sqrt{\alpha \sigma_{H_{0}}^{2}}} \exp \left[-\frac{1}{2}\left(\gamma-\frac{\beta^{2}}{\alpha}\right)\right]\left[1+\operatorname{erf}\left(\frac{\beta}{\sqrt{2 \alpha}}\right)\right]
$$

\section{$6 \chi^{2}$ Contours}

After applying the above equations and methods, we arrive at the results presented below.
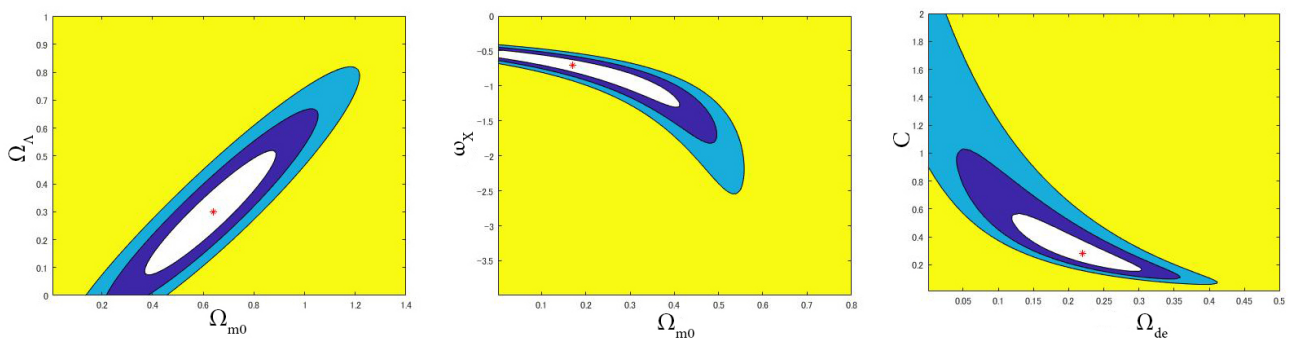

Figure 1. Constraint contours for the $\Lambda \mathrm{CDM}$ (left), XCDM (middle), Holographic model (right) for $H_{0}=68.0 \pm 2.0 \mathrm{kms}^{-1}$, from $H(z)$ data.

\section{Conclusions}

We are able to conclude that the cosmic chronometers is a very robust method of constraining dark energy. It gives very consistent constraints with prediction of spatially flat cosmological model with an energy budget dominated by a time independent cosmological constant, the standard $\Lambda C D M$ model, which has become tighter with the addition of newer, later data points. However, one must note that we arrive at similar $\chi^{2}$ values for all 3 models, meaning that we are unable to conclusively state which model is the best. After some exploration into whether conformal gravity is compatible with the Holographic model, we have found it to be incompatible due to the formation of singularities. 


\section{Future Works}

Data fitting using the Hubble parameter measurement is fundamentally different due to it being cosmology independent and its differential nature, and a combined constraint with other methods could prove fruitful. Other papers such as [6], [2] and [3] have done the combined constraints for the $\Lambda \mathrm{CDM}, \mathrm{XCDM}$ and $\phi \mathrm{CDM}$ models. However, a combined constraint for the Holographic model mentioned here has yet to be done. Even though the $\chi^{2}$ values for all 3 models that we tested are similar and there is a clear incompatibility with conformal gravity, Holographic Dark Energy remains a strong and unique contender to resolve the dark energy problem.

We thank Lim Yen Kheng for useful discussions.

\section{References}

[1] D. Stern, R. Jimenez, L. Verde, M. Kamionkowski, S.A. Stanford, Journal of Cosmology and Astroparticle Physics 2010, 008 (2010)

[2] M. Moresco, A. Cimatti, R. Jimenez, L. Pozzetti, G. Zamorani, M. Bolzonella, J. Dunlop, F. Lamareille, M. Mignoli, H. Pearce et al., Journal of Cosmology and Astroparticle Physics 2012, 006 (2012)

[3] M. Moresco, L. Pozzetti, A. Cimatti, R. Jimenez, C. Maraston, L. Verde, D. Thomas, A. Citro, R. Tojeiro, D. Wilkinson, Journal of Cosmology and Astroparticle Physics 2016, 014 (2016)

[4] E. Gaztanaga, A. Cabré, L. Hui, Monthly Notices of the Royal Astronomical Society 399, 1663 (2009)

[5] J. Simon, L. Verde, R. Jimenez, Phys. Rev. D 71, 123001 (2005)

[6] O. Farooq, D. Mania, B. Ratra, The Astrophysical Journal 764, 138 (2013)

[7] S. Wang, Y. Wang, M. Li, physrep 696, 1 (2017), 1612 .00345

[8] P.J.E. Peebles, The Astrophysical Journal 284, 439 (1984)

[9] B. Ratra, Phys. Rev. D 43, 3802 (1991)

[10] L. Susskind, Journal of Mathematical Physics 36, 6377 (1995), hep-th/9409089

[11] K. Ganga, B. Ratra, J.O. Gundersen, N. Sugiyama, The Astrophysical Journal 484, 7 (1997) 